\title{
Liposomal SN-38
}

National Cancer Institute

\section{Source}

National Cancer Institute. Liposomal SN-38. NCI Thesaurus. Code C95717.

The liposomal formulation of SN-38 (7-ethyl-10-hydroxy-camptothecin), a biologically active metabolite of the prodrug irinotecan, with potential antineoplastic activity. SN-38 binds to and inhibits topoisomerase I by stabilizing the cleavable complex between topoisomerase I and DNA, resulting in DNA breaks, inhibition of DNA replication, and apoptosis. SN-38 has been reported to exhibit up to 1,000-fold more cytotoxic activity against various cancer cells in vitro than irinotecan. The liposomal formulation of SN-38 increases the solubility of $\mathrm{SN}-38$, which is a relatively insoluble compound, and improves the pharmacodynamic profile as compared to SN-38 alone. 